\title{
Nonlinear Microscopy in Studying Extracellular Matrix State of the Urinary Bladder in Severe Complications after Radiation Therapy of Female Pelvic Tumors
}

DOI: $10.17691 / \mathrm{stm} 2017.9 .2 .02$

Received January 12, 2017

O.S. Streltsova, MD, DSc, Professor, Urology Department named after E.V. Shakhov';

A.V. Maslennikova, MD, DSc, Professor, Oncology, Radiotherapy, Radiodiagnosis Department

Professor, Biophysics Department²;

K.E. Yunusova, MD, PhD, Associated Professor, Department of Pathological Anatomy';

V.V. Dudenkova, Junior Researcher, Laboratory of Studying of Optical Structure of Biotissues,

Institute of Biomedical Technologies'; PhD Student, Department of General Physics, Radiophysics Faculty2

E.B. Kiseleva, PhD, Researcher, Laboratory of Studying of Optical Structure of Biotissues, Institute of Biomedical Technologies';

M.V. Kochueva, PhD Student, Oncology, Radiotherapy, Radiodiagnosis Department';

E.A. Tararova, MD, PhD, Oncologist3;

D.K. Malikov, Urologist";

A.S. Vorobieva, Urologist5;

V.N. Krupin, MD, DSc, Professor, Head of Urology Department named after E.V. Shakhov ${ }^{1}$

${ }^{1}$ Nizhny Novgorod State Medical Academy, 10/1 Minin and Pozharsky Square, Nizhny Novgorod, 603005,

Russian Federation;

'Lobachevsky State University of Nizhni Novgorod, 23 Prospect Gagarina, Nizhny Novgorod, 603950,

Russian Federation;

${ }^{3}$ Nizhny Novgorod Regional Oncologic Hospital, 190 Rodionova St., Nizhny Novgorod, 603126,

Russian Federation;

${ }^{4}$ Visus-1, 198B Rodionova St., Nizhny Novgorod, 603126, Russian Federation;

${ }^{5}$ N.A. Semashko Nizhny Novgorod Regional Hospital, 190 Rodionova St., Nizhny Novgorod, 603126,

Russian Federation

The study aimed to assess the characteristics of the urinary bladder extracellular matrix after radiotherapy for cervical cancer and hysterocarcinoma using nonlinear microscopy.

Materials and Methods. Two groups of patients were studied. The first group $(n=75)$ involved female patients with severe complications after combined radiotherapy for cervix cancer or endometrial cancer. Adverse events of urinary bladder developed within the period from a year to eleven years. The second group $(n=80)$ consisted of female patients suffering from chronic cystitis of bacterial etiology, their past history being over 3 years. We carried out a comparative analysis of the cystoscopic pattern of mucosa and the morphological analysis findings of urinary bladder bioptates.

For the first time there was studied the extracellular matrix state of the bladder connective tissue after radiotherapy by nonlinear microscopy carried out in the modes: second harmonic generation and two-photon excited autofluorescence to examine the state of collagen and elastin, respectively. To verify the obtained images we studied parallel histological sections stained by hematoxylin and eosin and picrosirius red.

Results. Nonlinear microscopy in radiation and chronic cystitis revealed similar inflammatory changes and tissue fibrosis. The intensity of radiation changes of the urinary bladder tissues depended directly on time after radiation, they being more intense and gross compared to those in chronic cystitis. Nonlinear microscopy enabled to reveal the difference in collagen and elastin structures after urinary bladder radiation damage of various severity. The structure of collagen fibers in II severity degree of radiation was preserved, the fibers being more packed, while III degree was characterized by marked disarrangement of collagen fibers.

Conclusion. The combination of optical methods (nonlinear microscopy combined with specific staining of histological preparations) enables to assess objectively structural changes of the urinary bladder extracellular matrix and determine the intensity of alterations after ionizing radiation.

The findings can serve as the basis to develop the approaches to visual and quantitative evaluation of the results of noninvasive optical techniques (e.g., polarization modifications of optical coherence tomography) to monitor radiation-induced damage in the urinary bladder.

Key words: extracellular matrix state; urinary bladder radiation damage; nonlinear microscopy; collagen; elastin.

For contacts: Olga S. Streltsova, e-mail: strelzova_uro@mail.ru 
Despite the development of conformal radiation techniques, the change of healthy tissues for malignancies resulting from radiotherapy still remains an unsolved problem in current radiation oncology. The latter are radiation injuries, which occur in a period of over 100 days after exposure, and frequently many years later after radiotherapy [1, 2]. They are of a great clinical significance, and their intensity can range from relatively insignificant functional damage to severe complications affecting the patients' life quality and occasionally they are life-threatening [3, 4]. The urinary bladder and rectum are the main organs at risk in radiotherapy for cancer of pelvic organs. Complications are caused by more aggressive chemotherapy and the combination of chemotherapy and radiation [5-8], as well as the use of nonstandard fractionation methods [9-11]. An increased risk of severe radiation-induced injuries develops in patients with chronic inflammatory diseases of the intestine and the urinary bladder [12, 13], diabetes mellitus [14], cardiovascular pathologies [15], sexually transmitted diseases in past history including HIV-infection [16] and systemic diseases of connective tissue [17].

The basic manifestation of late radiation alterations of the urinary bladder is pathological postradiation fibrosis due to vascular injuries, increased collagen synthesis (primarily, types I and III) and sequential activation of key fibrinogenic growth factors and cytokines, mainly TGFb1 and CTGF [18].

The study of morphofunctional changes of the urinary bladder when exposed to ionizing radiation has showed the late progressing organ damage to be manifested by the denudation of urothelial cells, the formation of ulcers and necroses, telangiectasia in mucosa and a submucous layer, fibrosis development $[19,20]$. These changes, as a rule, occur over a period of 2-3 years after radiation, and can result in a constant decrease of the bladder capacity, in some cases they requiring total cystectomy [21].

In most studies the data on the connective tissue bladder matrix state after radiation were obtained using standard histological techniques. The emergence of modern high-resolution techniques to image collagen and elastin: nonlinear microscopy in the modes of second harmonic generation (SHG) recording and twophoton excited autofluorescence (TPEF) will enable to assess these changes at a new level, make it possible to develop noninvasive optical tools to monitor radiationinduced damages to be used in clinical practice.

The aim of the investigation was to assess the possibilities to study the characteristics of the urinary bladder extracellular matrix after radiotherapy for cervical cancer and hysterocarcinoma using nonlinear microscopy.

Materials and Methods. To reveal the peculiarities of extracellular matrix damage of the urinary bladder after ionizing radiation and as a result of chronic inflammation, we studied two groups of patients. The first group $(n=75)$ involved female patients with severe complications after combined radiotherapy for cervix cancer or endometrial cancer. Adverse events of the urinary bladder developed within a period from a year to eleven years after radiotherapy. The second group $(n=80)$ consisted of female patients suffering from chronic cystitis of bacterial etiology, their past history being over 3 years.

The study was carried out in accordance with the Declaration of Helsinki (adopted in June 1964, Helsinki, Finland and revised in October 2000, Edinburgh, Scotland) and was approved by the Ethics Committee of Nizhny Novgorod State Medical Academy. All female patients gave their written informed consent.

All patients underwent the cystoscopic examination. 33 patients of group 1 and 28 patients of group 2 were performed the urinary bladder biopsy. The tissue samples of the urinary bladder for morphological examination were taken in posterior and lateral bladder walls, the tissues being both visually changed and visually unchanged. The Table shows the data on the patients, who underwent morphological examination of the urinary bladder mucosa.

Unchanged bladder samples (autopsy material, 3 samples) were used as controls.

Nonlinear microscopy and histological examination were performed according to the following technique: urinary bladder tissue samples for both investigations were fixed in $10 \%$ neutral formaldehyde solution for $24 \mathrm{~h}$ followed by paraffin embedding (Histomix extra medium; BioVitrum, Russia) and subsequent making paraffin blocks. Using a rotary microtome Leica 450 RM (Leica Microsystems, Germany) we made sections (10 and $0.1 \mu \mathrm{m}$ thick) of the obtained blocks. 10- $\mu \mathrm{m}$ samples were deparaffinized and studied by nonlinear microscopy without additional staining. To verify the obtained images, 0.1- $\mu \mathrm{m}$ samples prepared of the same blocks were stained by hematoxylin and eosin, as well as picrosirius red.

Nonlinear microscopy was performed on an inverted laser scanning microscope LSM Axiovert 510 Meta (Carl Zeiss, Germany). As a source of exciting radiation we used a short-pulse femtosecond laser MAI TAI HP (Spectra Physics, USA), the pulse repetition frequency being $80 \mathrm{~Hz}$ and pulse duration $100 \mathrm{fs}$. The excitement had wavelength $800 \mathrm{~nm}$, SHG signal from collagen structures was recorded in the range of $362-415 \mathrm{~nm}$ (imaging in green color), TPEF signal from elastic fibers was recorded in the range 480-555 nm (imaging in red color). Superimposition of SHG and TREF signals resulted in the third image, by which it was easier to assess the contribution of every component to a general signal from an object.

Stained histological specimens were studied using a microscope Leica DMLS (Leica Microsystems, Germany). Hematoxylin and eosin stained histological specimens were used to describe the nature of an inflammatory process and its intensity. Using these specimens we calculated the number of vessels of 
Characteristics of patients, who underwent a morphological study of the urinary bladder mucosa

\begin{tabular}{lcc}
\hline \multicolumn{1}{c}{ Parameters } & $\begin{array}{c}\text { Group 1 } \\
\text { (radiation cystitis) }\end{array}$ & $\begin{array}{c}\text { Group 2 } \\
\text { (chronic cystitis) }\end{array}$ \\
\hline Mean age (years), Me (min-max) & $54(36-86)$ & $51(25-75)$ \\
\hline Past history duration (years) & $1-11$ & More than 3 \\
\hline Cervix cancer/endometrial cancer (cases) & $27 / 6$ & - \\
\hline Cancer stage (I/I/IIII) & $1 / 24 / 8$ & - \\
\hline Combined radiotherapy/among them a splitting course (cases) & $19 / 4$ & - \\
\hline $\begin{array}{l}\text { Combined therapy: surgery + postoperative radiotherapy } \\
\text { (cases) }\end{array}$ & 14 & - \\
\hline Number of patients with II/III/IV degree of radiation cystitis & $15 / 14 / 4$ & $28 / 4 / 29$ \\
\hline $\begin{array}{l}\text { Number of biopsies/number of samples for nonlinear } \\
\text { microscopy/number of nonlinear microscopic }\end{array}$ & $33 / 9 / 86$ & \\
\hline
\end{tabular}

microcirculatory bloodstream per field of view, and for this purpose Avtandilov's morphometric eyepiece graticule with 289 intersections was used. The vessels were calculated at lens magnification 40, eye lens magnification 5 followed by the normalization of their number per unit area. Picrosirius red stained histological specimens were assessed by polarization microscopy that enabled to observe precisely the changes in collagen fibers [22].

\section{Results}

Cystoscopic examination. Cystoscopy revealed typical focal hyperemia on the posterior bladder wall in the female patients with complications after radiotherapy, sometimes there were petechial hemorrhages: in 47 cases $(62.7 \%)$. Hyperemia and edema in the triangle of the bladder occurred more frequently - in 54 cases $(72.0 \%)$. Severe (III and IV degrees) injury of the urinary bladder was found in 20 female patients, and in 14 of them we found subtotal telangiectasias, petechiae, erosive foci. The changes occurred mainly in the posterior wall area, the triangle of the urinary bladder ("hot" areas for radiotherapy) and the opening of ureters. Ulcers with necrosis occasionally encrusted with salts were found chiefly on the posterior wall. Four cases had vesicovaginal fistulas.

A visually unchanged bladder was revealed by cystoscopy in the female patients with chronic cystitis: 5 cases $(6.25 \%)$, marked vascular pattern was observed in 46 cases $(57.5 \%)$, edema and hyperemia in the triangle of the bladder against the marked vascular pattern was found in 19 cases $(23.75 \%$ ), and leukoplakia - in 37 cases $(46.25 \%)$.

Histological examination. The bladder tissue samples over a one-year period after radiotherapy were found to have fibrous changes of various intensity degrees. There was sclerosis of small vessels (their lumen diameter was significantly lower), as well as marked fibrosis of submucous structures. Almost in all the cases there was formed fibrous scar tissue in mucosa and submucosa of the urinary bladder against inflammatory infiltrates, primarily perivascular. In addition, picrosirius red staining revealed bright red and yellow luminescence of collagen fibers (I type collagen), mainly, around the vessels that is the sign of a starting vascular wall sclerosis (Figure 1 (a)). Elasticity loss of distributing vessels of microcirculatory bloodstream due to similar changes can result in one of the most threatening complications of radiation therapy of the small pelvis: heavy cystorrhagia. In most samples of the irradiated bladder at different postradiation periods, among fibrous areas we revealed small regions of granulation, it testifying the imperfection of regeneration processes against the proceeding alteration processes. Fibrous tissue was found in a muscular layer in the form of singular interlayers with focal atrophy of muscular elements. A histological specimen stained by picrosirius red showed closely packed thick collagen fiber bundles forming fibrous tissue both in a submucous layer and a muscular layer. Red-orange fiber color suggests type I collagen prevailing, fiber bundles having parallel packing (Figure 1 (c)).

A comparative microscopic analysis of visually unchanged and visually altered bladder areas in radiation cystitis showed the tissue damage degree in the samples to be different. The bioptates from hemorrhage areas on the posterior wall were found to have erythrodiapedesis. Surface urothelium was of various thickness: there were atrophy, focal hyperplasia areas, and some places with marked dystrophic changes. Subepithelial tissue was revealed to have acute hyperemia, marked edema, hemorrhage (Figure 2 (a), yellow arrow), perivascular focal infiltrates of lymphocytes, plasma cells, histiocytes (Figure 2 (a), black arrows) indicating the presentation of exudative infiltrative inflammation. It is essential that the mucosa of visually unchanged lateral bladder walls was also 

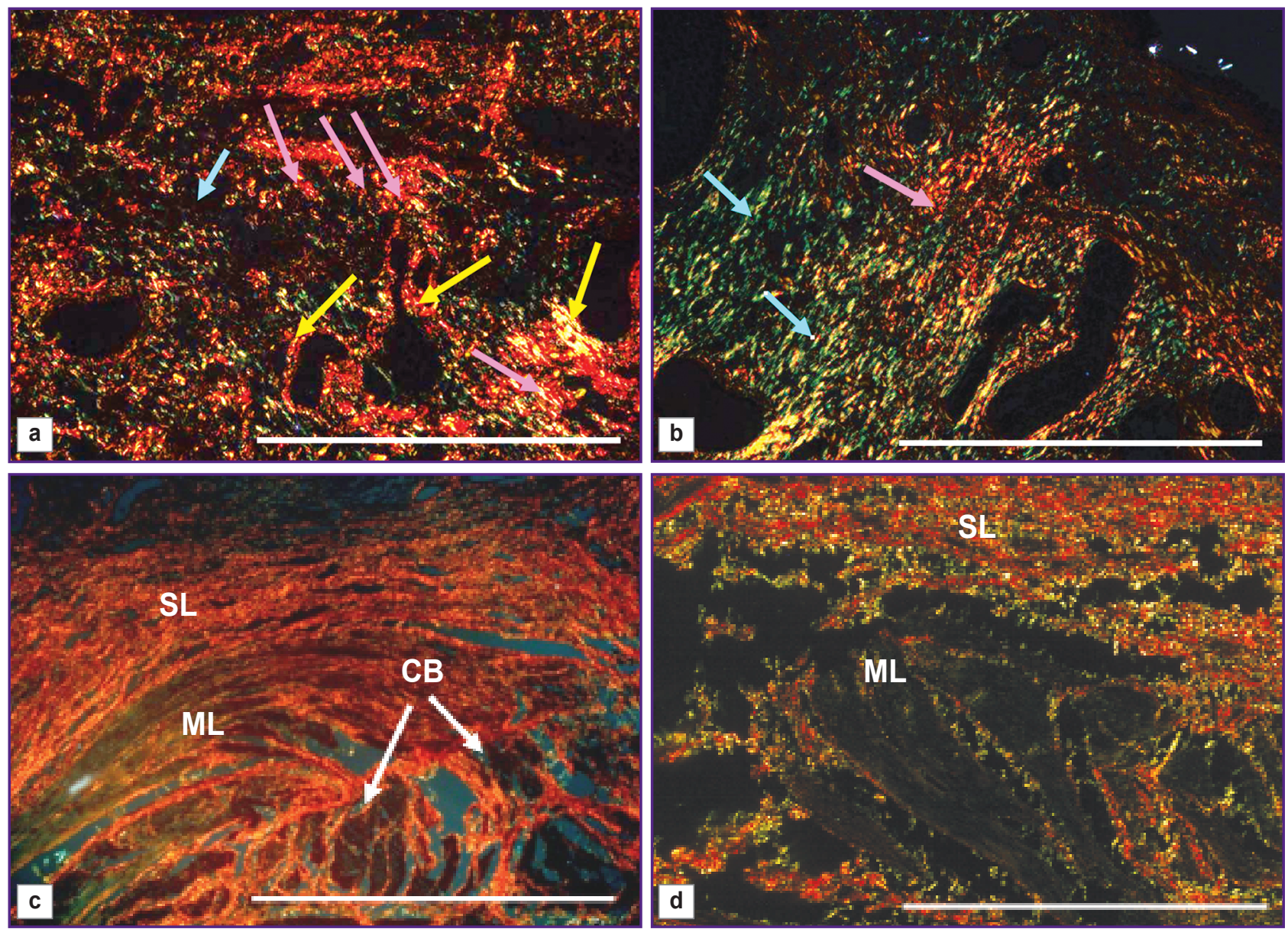

Figure 1. Comparative histological analysis of the bladder extracellular matrix in radiation (a), (c) and chronic (b), (d) cystitis:

in radiation cystitis, in a submucous layer (a) there was revealed nascent sclerosis of vascular wall (yellow arrows), fibrosis areas (pink arrows) and small regions of granulation tissue (a light-blue arrow); in a muscular layer (c) there can be observed closely packed thick bundles of collagen fibers forming fibrosis tissue in combination with focal atrophy of muscular elements; in chronic cystitis, in a submucous layer (b) fibrosis areas (thickened collagen fibers of red-orange color) were determined only on some regions (pink arrow) against the prevailing inflammatory processes (thin collagen fibers of green color) (light-blue arrows); in a muscular layer (d) no connective tissue accrementition was found; picrosirius red staining, a microphoto was taken in polarized light. SL: submucous layer; ML: muscular layer; CB: collagen bundles; bar: $1 \mathrm{~mm}$
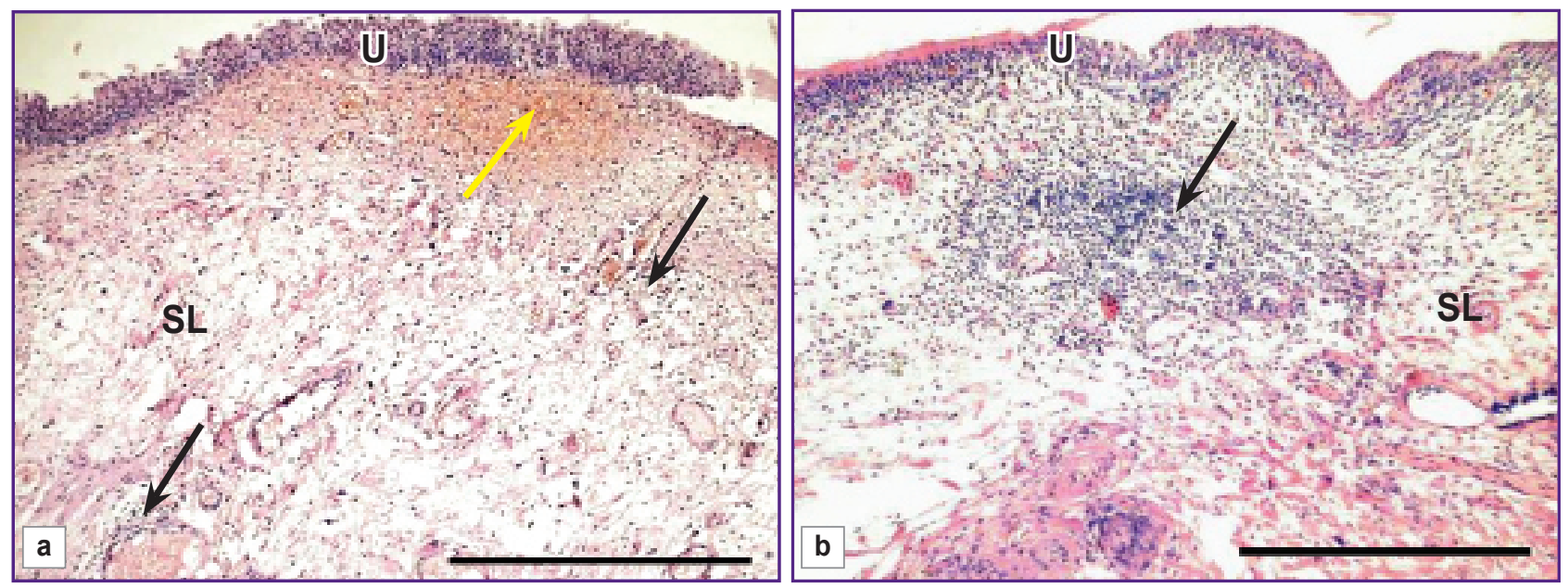

Figure 2. The bladder wall in radiation cystitis:

comparative histological analysis of visually changed (a) and visually unchanged (b) areas; hematoxylin and eosin staining. U: urothelium, SL: submucous layer; black arrows indicate infiltrates; a yellow arrow indicated hemorrhagia; bar: $1 \mathrm{~mm}$ 
found to have similar alterations though in a lesser degree. Lymphocytic infiltration foci were revealed in the submucous layer of several specimens (Figure 2 (b), arrow) that is typical for a chronic inflammatory process.

The bladder tissue samples of the patients with chronic cystitis showed diverse alterations of the surface epithelium in the form of atrophy areas, and occasionally - hyperplasia, acanthosis with the formation of Brunn's foci, irregular enlargement of fibrous tissue, which includes the vascular walls, inflammatory lymphocytoplasmocytic infiltrates occurring primarily in wall intima. Red-orange staining of collagen fibers consistent with fibrosis foci (Figure 1 (b), pink arrow) was determined on some areas against the prevailing inflammatory processes (thin collagen fibers of green color) (Figure 1 (b), light-blue arrows). No connective tissue enlargement was observed in a muscular layer (Figure $2(d)$ ).

Thus, in chronic cystitis lasting for a period of over three years and in radiation cystitis for more than a
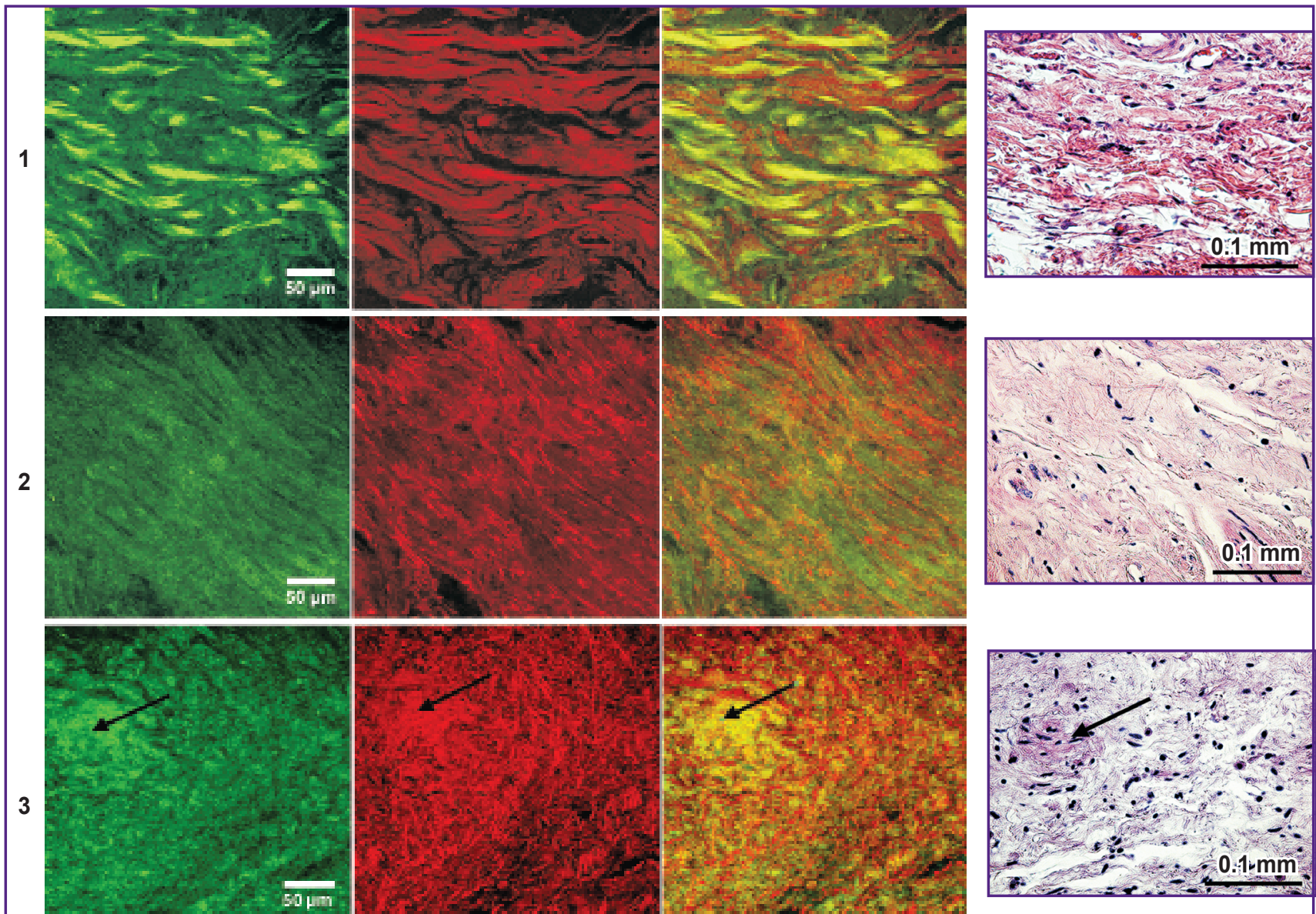

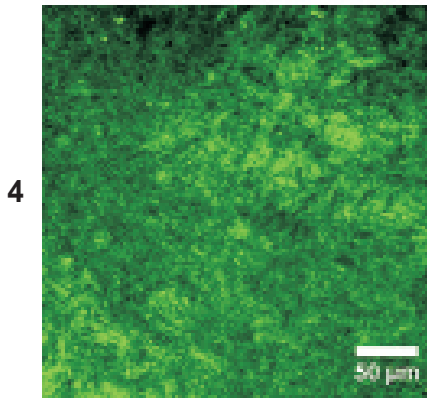

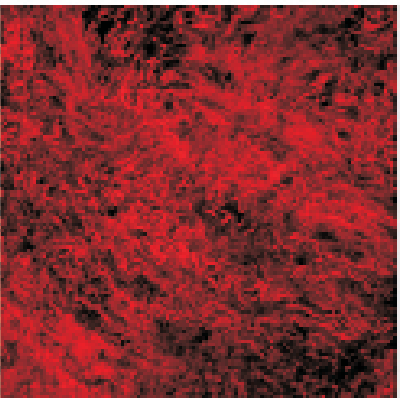

b

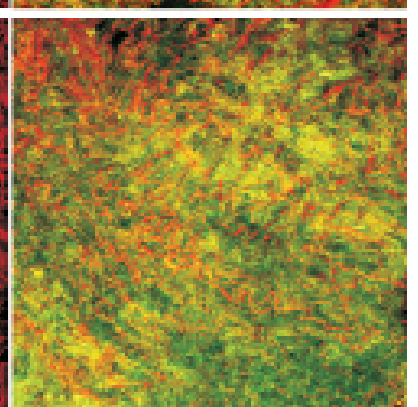

C
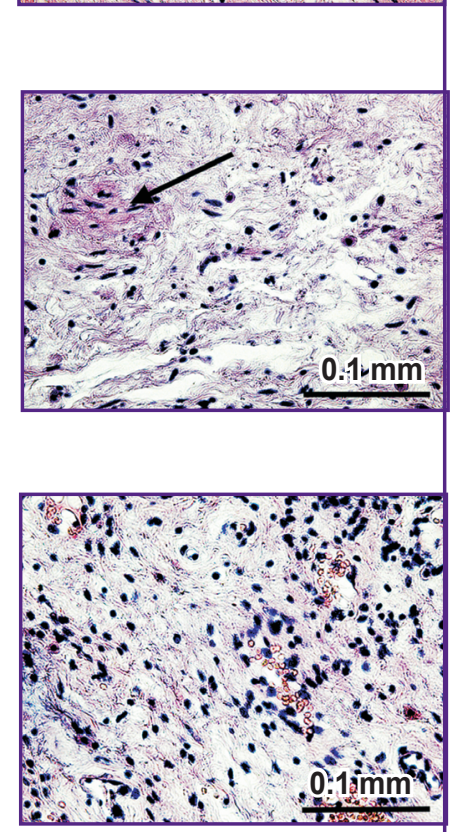

d

Figure 3. Nonlinear microscopy and histological examination of the bladder connective tissue:

norm (row 1), radiation cystitis, II severity degree (row 2), radiation cystitis, III severity degree (row 3), chronic cystitis (row 4); nonlinear microscopic image in SHG mode (a), TPEF (b), combined SHG+TPEF image (c); corresponding histological specimens (d), hematoxylin and eosin staining; a black arrow indicates the focus of structureless tissue with a high SHG signal (a)-(c), (row 3 ) that can be consistent with progressing hyalinosis (d), (row 3 ) 
year, marked changes form in a submucous layer of the urinary bladder. After radiotherapy the marked fibrous areas are found in a muscular layer as well. There is a tendency for a reduced number of vessels of microvasculature per a tissue area unit in radiation complications compared to chronic cystitis: $62 \pm 12$ per $1 \mathrm{~mm}^{2}$ and $73 \pm 8$ per $1 \mathrm{~mm}^{2}$, respectively, though no significant differences were found. The main difference in the bladder tissue state in radiation damage was more significant structure damage in all bladder wall layers.

Nonlinear microscopy. The use of the technique enabled to study in more detail the bladder connective tissue matrix state. Collagen fibers in healthy urinary bladder are clearly seen, tortuous, loosely packed and have elastic fibers. In some places there are bundles of collagen fibers, 10-15 $\mu \mathrm{m}$ in diameter (Figure 3 (a)-(c), row 1).

In radiation damage of the bladder, the extracellular matrix state depended on the severity of radiotherapy side effects. In II severity degree, the bundles of collagen fibers have sharp contours, closely packed, with preferential direction (Figure 3 (a), row 2). Elastic fibers are thin, their direction is similar to that of collagen bundles (Figure 3 (b), row 2). In III degree of radiation damage, collagen fibers have fuzzy contour, desorganized, highly crimpled, without preferential direction (Figure 3 (a), row 3). Elastic fibers are thin, clearly-cut, without privileged direction, having mosaically arranged highly crimpled areas. There is the loss of crimpled form of packing typical for healthy elastic fibers (Figure 3 (b), row 3 ). In the vast majority of studied samples with severe radiation damage we revealed the lesions of structureless tissue with high SHG signal (Figure 3 (a), row 3, arrow) indicating a high degree of anisotropy and density, though without organization signs. Such peculiarity of SHG signal recording can be consistent with hyalinosis, however, the comparison of the described areas with histological specimens revealed that when hematoxylin and eosin stained they are not clearly differentiated against fibrous tissue (Figure 3 (d), row 3, arrow).

Nonlinear microscopy of the urinary bladder in chronic cystitis revealed no such foci. The distribution of SHG signal from collagen image visualizes fuzzy, disorganized, thick bundles without preferential direction (Figure 3 (a), row 4). Histology revealed fibrous tissue with inflammatory infiltrates (Figure $3(\mathrm{~d})$, row 4). Elastic fibers are thin, and their number is significantly reduced (Figure $3(\mathrm{~b})$, row 4 ) that can have an impact on the urinary bladder functionality.

Discussion. Basic morphofunctional peculiarities characterizing bladder tissue after the effect of ionizing radiation on the tumors of female reproductive system were revealed experimentally over 30 years ago: radiation fibrosis, severe epithelial hyperplasia, which interchanges denudation lesions, focal necrosis of smooth muscle cells and their substitution by collagen fibers due to hyperplasia of the latter $[20,23]$. Further studies [24] showed early and late radiation damage to be based on impaired urothelial barrier due to the death of highly specialized polyploid surface cells. The progression of impaired microcirculation was also shown to play one of key roles in radiation damage of the urinary bladder $[21,25]$. The mentioned studies were carried out on animal models under single exposure irradiation that prevents from extrapolating the findings to the urinary bladder state of patients with radiotherapy risk.

The specific character of our work is the use of several techniques to assess the state of collagen- and elastincontaining bladder structures. Standard histological examination with hematoxylin and eosin staining revealed urothelial atrophy and focal hyperplasia, in underlying connective tissue stroma - hyperemia, marked edema, hemorrhage, focal infiltrates from lymphocytes, plasma cells, histiocytes that is consistent with the alterations described in the study [24]. Picrosirius red staining enables to reveal collagen fibers of different types making it possible to study in more detail their structural and three-dimensional organization, and helps to interpret the nonlinear microscopic images.

Nonlinear microscopy with SHG signal detection enables to study in detail the state of collagencontaining structures of biological tissues without additional staining. The previous studies carried out using this technique [26, 27] showed the decrease of SHG signal intensity to be the main manifestation of radiation damage of collagen at an early postradiation period. For the first time in medical practice, the present investigation using nonlinear microscopy studied the peculiarities of the state of collagen-containing bladder structures after ionizing radiation compared to chronic inflammation.

The basic manifestation of the bladder exposure sequelae is the formation of fibrosis, which is more marked compared to the alterations occurring in chronic cystitis. At the late postradiation stages fibrosis manifestations increase compared to the early period that is consistent with literature data. We found the difference in the state of collagen and elastin structures in bladder radiation damage of various severity degrees, and the differences cannot be revealed using standard histological examination and specific staining of collagen by picrosirius red.

The basic manifestation of the II degree of radiation complications was the formation of more dense and "rigid" ordered structure of collagen fibers that is the evidence of prevailing neocollagenesis processes, i.e. "classical" fibrosis formation. The clinical presentation consistent with the III degree of radiation side effects had marked disorganization of collagen-containing structures manifesting itself as diffuse SHG signal decrease, fuzzy and blurring fiber structure. Moreover, nonlinear microscopic images showed small foci of structureless tissue with a high SHG signal, the interpretation of the changes using standard histological examination 
failed. Depolymerization of glycosaminoglycan, the breaking of collagen fibers and their saturation with plasma proteins are known to occur during connective tissue disorganization. According to Pavlova et al. [28], collagen forms dense insoluble compounds with fibrinogen and other substances. It can be assumed that connective tissue destruction areas with developing fibrinoid necrosis manifest themselves in this way on SHG-images [29].

The findings can serve as the basis to develop new approaches to visual and quantitative evaluation of the bladder connective tissue matrix state after the radiation impact using noninvasive optical techniques, e.g., crosspolarization optical coherence tomography [30].

Conclusion. The use of nonlinear microscopy enables to study in detail collagen structure alterations in radiation and chronic cystitis, and reveal the differences in connective tissue matrix state in case of nonspecific inflammation and radiation-induced damage. Nonlinear microscopy makes it possible to reveal the peculiarities of connective tissue condition depending on the severity degree of the radiation injury that was impossible using routine methods of histological examination.

Study Funding. The work was supported by a grant Russian Foundation for Basic Research No.16-0700655.

Conflicts of Interest. The authors have no conflicts of interest related to the present study.

\section{References}

1. Dörr W. Radiation effect in normal tissue - principles of damage and protection. Nuklearmedizin 2010; 49(Suppl 1): S53-S58.

2. Dörr W., Hendry J.H. Consequential late effects in normal tissues. Radiother Oncol 2001; 61(3): 223-231, https:// doi.org/10.1016/s0167-8140(01)00429-7.

3. Dörr W. Pathogenesis and repair of normal tissue damage. Radiother Oncol 2011; 99: S135, https://doi. org/10.1016/s0167-8140(11)70460-1.

4. Cancer Therapy Evaluation Program. Common terminology criteria for adverse events (CTCAE) (version 4.03). 2010. URL: https://evs.nci.nih.gov/ftp1/CTCAE/ CTCAE_4.03_2010-06-14_QuickReference_5x7.pdf.

5. Stone H.B., Coleman C.N., Anscher M.S., McBride W.H. Effects of radiation on normal tissue: consequences and mechanisms. Lancet Oncol 2003; 4 (9): 529-536, https://doi. org/10.1016/s1470-2045(03)01191-4.

6. Haie-Meder C., de Crevoisier R., Bruna A., Lhommé C., Pautier P., Morice P., Castaigne D., Bourhis J. Concomitant chemoradiation in patients with cervix cancer. Bull Cancer 2005; 92(12): 1032-1038.

7. Tan L.T., Zahra M. Long-term survival and late toxicity after chemoradiotherapy for cervical cancer - the addenbrooke's experience. Clin Oncol 2008; 20(5): 358-364, https://doi.org/10.1016/j.clon.2008.03.001.

8. Boltenko A.I. Modern treatment of cervix uteri carcinoma, new methods of combined therapy. Vestnik Rossiyskogo nauchnogo tsentra rentgenoradiologii 2008; 8. URL: http://vestnik.rncrr.ru/vestnik/v8/papers/boltenko_v8.htm.
9. Moonen L., van der Voet H., Horenblas S., Bartelink H. A feasibility study of accelerated fractionation in radiotherapy of carcinoma of the urinary bladder. Int $J$ Radiat Oncol Biol Phys 1997; 37(3): 537-542, https://doi.org/10.1016/s03603016(96)00541-X.

10. Horwich A., Dearnaley D., Huddart R., Graham J., Bessell E., Mason M., Bliss J. A randomised trial of accelerated radiotherapy for localised invasive bladder cancer. Radiother Oncol 2005; 75(1): 34-43, https://doi.org/10.1016/j. radonc.2004.11.003.

11. Soete G., Arcangeli S., De Meerleer G., Landoni V., Fonteyne V., Arcangeli G., De Neve W., Storme G. Phase II study of a four-week hypofractionated external beam radiotherapy regimen for prostate cancer: report on acute toxicity. Radiother Oncol 2006; 80(1): 78-81, https://doi. org/10.1016/j.radonc.2006.06.005.

12. Willett C.G., Ooi C.J., Zietman A.L., Menon V., Goldberg S., Sands B.E., Podolsky D.K. Acute and late toxicity of patients with inflammatory bowel disease undergoing irradiation for abdominal and pelvic neoplasms. Int $J$ Radiat Oncol Biol Phys 2000; 46(4): 995-998, https://doi.org/10.1016/ s0360-3016(99)00374-0.

13. Kaprin A.D., Titova V.A., Kreynina Yu.M., Kostin A.A. Urologicheskie oslozhneniya $v$ onkoginekologicheskoy praktike: diagnostika, interventsionnaya $i$ konservativnaya korrektsiya [Urologic complications in oncogynecological practice: diagnostics, interventional and conservative correction]. Moscow; 2011; 168 p.

14. Herold D.M., Hanlon A.L., Hanks G.E. Diabetes mellitus: a predictor for late radiation morbidity. Int $J$ Radiat Oncol Biol Phys 1999; 43(3): 475-479, https://doi.org/10.1016/ s0360-3016(98)00460-x.

15. Hamstra D.A., Stenmark M.H., Ritter T., Litzenberg D., Jackson W., Johnson S., Albrecht-Unger L., Donaghy A., Phelps L., Blas K., Halverson S., Marsh R., Olson K., Feng F.Y. Age and comorbid illness are associated with late rectal toxicity following dose-escalated radiation therapy for prostate cancer. Int J Radiat Oncol Biol Phys 2013; 85(5): 1246-1253, https:// doi.org/10.1016/j.ijrobp.2012.10.042.

16. Eifel P.J., Jhingran A., Bodurka D.C., Levenback C., Thames $\mathrm{H}$. Correlation of smoking history and other patient characteristics with major complications of pelvic radiation therapy for cervical cancer. J Clin Oncol 2002; 20(17): 36513657, https://doi.org/10.1200/jco.2002.10.128.

17. Shadad A.K. Gastrointestinal radiation injury: prevention and treatment. World J Gastroenterol 2013; 19(2): 199-208, https://doi.org/10.3748/wjg.v19.i2.199.

18. Yarnold J., Brotons M.-C. Pathogenetic mechanisms in radiation fibrosis. Radiother Oncol 2010; 97(1): 149-161, https://doi.org/10.1016/j.radonc.2010.09.002.

19. Antonakopoulos G.N., Hicks R.M., Hamilton E., Berry R.J. Early and late morphological changes (including carcinoma of the urothelium) induced by irradiation of the rat urinary bladder. Br J Cancer 1982; 46(3): 403-416, https://doi. org/10.1038/bjc.1982.217.

20. Stewart F.A. Mechanism of bladder damage and repair after treatment with radiation and cytostatic drugs. $\mathrm{Br} \mathrm{J}$ Cancer Suppl 1986; 7: 280-291.

21. Stewart F.A.., Akleyev A.V., Hauer-Jensen M., Hendry J.H., Kleiman N.J., Macvittie T.J., Aleman B.M., Edgar A.B., Mabuchi K., Muirhead C.R., Shore R.E., Wallace W.H. ICRP Publication 118: ICRP statement on tissue reactions and early and late effects of radiation in normal 
tissues and organs - threshold doses for tissue reactions in a radiation protection context. Ann ICRP 2012; 41(1-2): 1-322, https://doi.org/10.1016/j.icrp.2012.02.001.

22. Lattouf R., Younes R., Lutomski D., Naaman N., Godeau G., Senni K., Changotade S. Picrosirius red staining: a useful tool to appraise collagen networks in normal and pathological tissues. J Histochem Cytochem 2014; 62(10): 751-758, https://doi.org/10.1369/0022155414545787.

23. Antonakopoulos G.N., Hicks R.M., Berry R.J. The subcellular basis of damage to the human urinary bladder induced by irradiation. J Pathol 1984; 143(2): 103-116, https:// doi.org/10.1002/path.1711430205.

24. Jaal J., Dörr W. Radiation-induced damage to mouse urothelial barrier. Radiother Oncol 2006; 250-256, https://doi. org/10.1016/j.radonc.2006.07.015.

25. Jaal J., Dörr W. Radiation effects in mouse urinary bladder: changes in vascular volume. Radiother Oncol 2003; 67(Suppl 1): S8, https://doi.org/10.1016/s01678140(03)80560-1.

26. Maslennikova A., Kochueva M., Ignatieva N., Vitkin A., Zakharkina O., Kamensky V., Sergeeva E., Kiseleva E.,
Bagratashvili V. Effects of gamma irradiation on collagen damage and remodeling. Int J Radiat Biol 2015; 91(3): 240247, https://doi.org/10.3109/09553002.2014.969848.

27. Kuznetsov S.S., Dudenkova V.V., Kochueva M.V., Kiseleva E.B., Ignatieva N.Yu., Zakharkina O.L., Sergeeva E.A., Babak K.V., Maslennikova A.V. Multiphoton microscopy in the study of morphological characteristics of radiation-induced injuries of the bladder. Sovremennye tehnologii $v$ medicine 2016; 8(2): 31-39, https://doi. org/10.17691/stm2016.8.2.04.

28. Pavlova V.N., Kop'eva T.N., Slutskiy L.I., Pavlov G.G. Khryashch [Cartilage]. Moscow: Meditsina; 1988; 320 p.

29. Gistologiya [Histology]. Pod red. Ulumbekova E.G., Chelysheva Yu.A. [Ulumbekov E.G., Chelyshev Yu.A. (editors)]. Moscow: GEOTAR-Med; 2001; 670 p.

30. Kiseleva E., Kirillin M., Feldchtein F., Vitkin A., Sergeeva E., Zagaynova E., Streltzova O., Shakhov B., Gubarkova E., Gladkova N. Differential diagnosis of human bladder mucosa pathologies in vivo with cross-polarization optical coherence tomography. Biomed Opt Express 2015; 6(4): 1464-1476, https://doi.org/10.1364/boe.6.001464. 